\title{
PRÁTICA ALIMENTAR E DE ATIVIDADE FÍSICA EM ADOLESCENTES OBESOS DE ESCOLAS PÚBLICAS E PRIVADAS
}

\author{
Feeding behavior and physical activity in obese adolescents of \\ public and private schools \\ Práctica alimentaria y de actividad física en adolescentes \\ obesos de escuelas públicas y privadas
}

\begin{abstract}
RESUMO
Objetivo: Avaliar a prática alimentar e o nível de atividade física em adolescentes obesos de escolas públicas (EPu) e privadas (EPr) de Fortaleza-CE. Métodos: Estudo transversal realizado com 272 adolescentes de Epu e EPr, no período de abril de 2007 a maio de 2009. Aplicou-se um questionário contendo dados de identificação (idade, sexo), antropométricos (peso e altura), situação escolar, prática alimentar e nível de atividade física. Resultados: Dos avaliados, observou-se que $160(58,8 \%)$ eram do sexo feminino, com idade média de 16,5 anos $( \pm 1,31)$. Houve prevalência da obesidade moderada, com 85,5\%, $(n=171)$ na EPu e $76,4 \%,(n=55)$ na EPr. Não se constatou diferença entre o grau de obesidade e a rede escolar. Sobre a prática alimentar, 70,2\% $(\mathrm{n}=190)$ consumiam menos de cinco refeições ao dia e $56,3 \%(n=153)$ não faziam o café da manhã, não tendo diferença entre as redes escolares. O consumo de frutas $(10 \% ; n=27)$ e vegetais $(14,1 \% ; n=38)$ foi baixo. Os estudantes das EPr consumiam mais refrigerantes $(41,7 \% ; n=30)$ do que os da $\mathrm{EPu}(28,5 \% ; n=57 ; \mathrm{p}=0,03)$. Constatou-se, que 47\% (n=93) dos alunos da EPu e que 32,4\% (n=27) da EPr não consumiam fast-food $(\mathrm{p}=0,02)$. Identificou-se que os adolescentes das EPu eram mais ativos do que os das $\operatorname{EPr}(77 \% ; n=153$ versus $54,2 \% ; n=40 ; p=0,0002)$, mas essa diferença não mostrou associação com o grau de obesidade $(\mathrm{p}=0,88)$. Conclusão: Os adolescentes das escolas públicas e particulares apresentavam prática alimentar inadequada, havendo poucas diferenças entre eles. Os jovens das EPu apresentaram-se mais ativos que os das EPr, entretanto, este nível de atividade física não influenciou no grau de obesidade.
\end{abstract}

Descritores: Obesidade; Adolescentes; Comportamento alimentar; Atividade motora.

\section{ABSTRACT}

Objective: To evaluate the quality of food and level of physical activity in obese adolescents from public (PuS) and private (PrS) schools of Fortaleza-CE. Methods: Cross-sectional study held with 272 adolescents from PuS and PrS in the period from April 2007 to May 2009. A questionnaire was applied containing identification (age, gender) and anthropometric (weight and height) data, school situation, feeding behavior and physical activity level. Results: It was observed that 160 (58.8\%) of the adolescents were female with a mean age of 16.5 years $( \pm 1.31)$. There was prevalence of moderate obesity, with $85.5 \%(n=171)$ in PuS and 76.4\% (n=55) in PrS. No difference was found between the obesity degree and the school network. Regarding feeding behavior, $70.2 \%(n=190)$ consumed less than five meals a day and $56.3 \%(n=153)$ had no breakfast, without differences between the school networks. The consumption of fruits $(10 \%, n=27)$ and vegetables $(14.1 \%, n=38)$ was low. Students of $\operatorname{PrS}$ consumed more soft drinks $(41.7 \%, n=30)$ than those of the PuS $(28.5 \%, n=57, p=0.03)$. Among students of the PuS and PrS, those who did not consume fast food represented $47 \%$ $(n=93)$ and $32.4 \%(n=27)$, respectively $(p=0.02)$. It was identified that the PuS adolescents were more active than those of $\operatorname{PrS}(77 \%, n=153$ versus $54.2 \%, n=40 ; p=0.0002)$, but this difference was not associated with the degree of obesity $(p=0.88)$. Conclusion: The adolescents of both the PuS and PrS had inadequate feeding behavior, with little differences between them. The adolescents of the PuS were more active than the ones of PrS, although this level of physical activity did not influence the obesity degree.

Descriptors: Obesity; Teens; Feeding Behavior; Motor activity.
Artigo Original
1) Universidade de Fortaleza - UNIFOR Fortaleza (CE) - Brasil

2) Instituto Federal de Educação, Ciência e Tecnologia do Ceará - IFCE - Fortaleza (CE) - Brasil

3) Universidade Estadual do Ceará - UECE - Fortaleza (CE) - Brasil

4) Universidade Federal do Ceará - UFC Fortaleza (CE) - Brasil
Recebido em: 13/10/2012 Revisado em: 12/06/2013 Aceito em: 02/12/2013 


\section{RESUMEN}

Objetivo: Evaluar la práctica alimentaria y el nivel de actividad física en adolescentes obesos de escuelas públicas (EPu) y privadas (EPr) de Fortaleza-CE. Métodos: Estudio trasversal realizado con 272 adolescentes de EPu y EPr entre abril de 2007 y mayo de 2009. Se aplicó un cuestionario con datos de identificación (edad y sexo), antropométricos (peso y altura), situación en la escuela, práctica alimentaria y nivel de actividad física. Resultados: De los adolescentes evaluados se observó que $160(58,8 \%)$ eran del sexo femenino con edad media de 16,5 años $( \pm 1,31)$. Hubo prevalencia de obesidad moderada en el $85,5 \%$ $(n=171)$ de la EPu y el 76,4\% (n=55) de la EPr. No se constató diferencia en el grado de obesidad y la red escolar. Respecto la práctica alimentaria, el 70,2\% (n=190) consumía menos de cinco comidas al día y el 56,3\% $(n=153)$ no desayunaba, no habiendo diferencia en las redes escolares. Fue bajo el consumo de frutas $(10 \% ; n=27)$ y vegetables $(14,1 \% ; n=38)$. Los estudiantes de las EPr consumían más refrescos $(41,7 \% ; n=30)$ que los de la EPu (28,5\%; $n=57 ; p=0,03)$. Se constató que el 47\% (n=93) de los alumnos de la EPu y el 32,4\% (n=27) de la EPr no consumian fast-food ( $p=0,02)$. Se identificó que los adolescentes de las EPu eran más activos que los de las $E P r(77 \% ; n=153$ versus 54,2\%; $n=40 ; p=0,0002)$, pero esa diferencia no mostró asociación con el grado de obesidad $(p=0,88)$. Conclusión: Los adolescentes de las escuelas públicas y privadas presentaron hábitos alimentarios inadecuados con poca diferencia entre ellos. Los jóvenes de las EPu se presentaron más activos que los de las EPr, sin embargo, este nivel de actividad física no ha influenciado en el grado de la obesidad.

Descriptores: Obesidad, Adolescente, Conducta Alimentaria, Actividad Motora.

\section{INTRODUÇÃO}

A obesidade é uma doença crônica caracterizada por um acúmulo anormal ou excessivo de gordura no organismo, podendo acarretar diversas consequências maléficas, como as doenças cardiovasculares, o Diabetes Mellitus, os distúrbios metabólicos e endócrinos, a apnéia do sono, as osteoartrites, certos tipos de câncer e vários problemas psicológicos ${ }^{(1,2)}$. Atualmente, é considerada um dos principais problemas de saúde pública, tanto nos países desenvolvidos quanto naqueles em desenvolvimento, sendo uma doença universal e de prevalência crescente ${ }^{(3,4)}$.

Fatores sociais, comportamentais, ambientais, culturais, psicológicos, metabólicos, bem como genéticos, podem acarretar um acúmulo excessivo de gordura corporal, sendo proveniente de um desequilíbrio energético prolongado ${ }^{(5)}$. Entre os ambientais, enfatiza-se a ingestão energética excessiva e a atividade física diminuída, sendo esses fatores etiológicos mais prevalentes na obesidade, podendo ter início em qualquer época da vida ${ }^{(6)}$.
A obesidade torna-se ainda mais problemática quando surge ou se agrava na adolescência, uma fase da vida onde ocorrem intensas transformações no processo de crescimento e desenvolvimento, particularmente, em razão da predominância de atividades de lazer sedentárias e práticas alimentares inadequadas, tais como o consumo de lanches hipercalóricos em substituição às principais refeições e elevada ingestão de alimentos ricos em carboidratos refinados e gordura saturada, isto é, os fast foods $s^{(7)}$. Além disso, adolescência representa um período crítico para o desenvolvimento da adiposidade, já que o risco de se tornar adulto com sobrepeso ou obeso aumenta com a idade ${ }^{(8)}$.

No Brasil, os resultados da Pesquisa de Orçamentos Familiares (POF) realizada pelo Instituto Brasileiro de Geografia e Estatística (IBGE) nos anos de 2008-2009 apontam mudanças no hábito alimentar dos brasileiros, onde foi visualizada uma tendência de crescimento na aquisição de alimentos ricos em lipídios e elevação dos carboidratos simples, acompanhada de redução na aquisição de alimentos fonte de carboidratos complexos ${ }^{(9)}$.

Comparando os dados do Estudo Nacional da Despesa Familiar (ENDEF) (1974-1975) com os do POF (2008 -2009), constatou-se, na faixa etária de dez a dezenove anos entre os homens, que a prevalência de excesso de peso passou de 3,7\% para $21,7 \%$; já nas mulheres, o crescimento do excesso de peso foi de 7,6\% para 19,4\%. Em relação à obesidade, essa também teve um aumento de $0,4 \%$ para $5,9 \%$ no sexo masculino e de $0,7 \%$ para $4 \%$ no feminino, tendo crescimento ascendente. No mesmo período, o déficit de peso reduziu de $10,1 \%$ para $3,7 \%$ nos homens e de $5,1 \%$ para 3,0\% nas mulheres ${ }^{(9)}$.

A análise desses resultados pode comprovar, assim, a transição nutricional e a magnitude do problema, mostrando carências, ainda existentes, e os excessos em ascensão ${ }^{(10)}$. É relevante enfatizar a maneira como está acontecendo a transição nutricional no Brasil. Deve ser feito um alerta, pois os jovens têm mais chances de ser obesos em locais onde a transição nutricional ocorreu de forma rápida ${ }^{(11)}$.

É importante que, na adolescência, haja a prevenção da obesidade pela prática de atividade física e pelo controle alimentar. Essas medidas podem contribuir para a melhoria do perfil lipídico e metabólico ${ }^{(12)}$. Devido a obesidade ter causas multifatoriais, a eficácia na prevenção e no tratamento é igualmente complexa, sem apresentar método único, sendo necessário uma intervenção mais abrangente com uma equipe multidisciplinar, com a finalidade de promover um balanço energético negativo, por meio da diminuição da ingestão calórica e do aumento da atividade física, para obter uma perda de peso gradual ${ }^{(8)}$.

Dessa forma, objetivou-se, no presente estudo, avaliar a prática alimentar e o nível de atividade física em 
adolescentes obesos de escolas públicas $(\mathrm{EPu})$ e privadas (EPr) de Fortaleza.

\section{MÉTODOS}

Trata-se de um estudo observacional, de caráter transversal e analítico ${ }^{(13)}$, desenvolvido entre abril de 2007 a maio de 2009.

A pesquisa teve representatividade da população de adolescentes entre 15 e 19 anos, haja vista que a taxa de escolarização bruta no ensino médio da cidade de Fortaleza, correspondendo à faixa etária da pesquisa, era de aproximadamente $90 \%{ }^{(14)}$. O número de estudantes avaliados em cada Secretaria Executiva Regional (SER) foi estabelecido pela proporcionalidade da população daquela SER com o número de alunos determinado previamente para compor a amostra.

Vale ressaltar que foi escolhida essa faixa etária dos adolescentes por já terem passado pelas marcantes modificações da adolescência, estando no período póspuberal. Desse modo, os parâmetros antropométricos de composição corporal são mais estáveis, proporcionando uma avaliação mais uniforme ${ }^{(15)}$.

Para a realização do cálculo amostral, utilizou-se o programa Epi-Info versão 6.04, tendo sido considerado uma prevalência em adolescentes obesos com síndrome metabólica de $32 \%{ }^{(16)}$. O nível de significância foi fixado em $5 \%$ e um erro amostral absoluto foi de $6 \%$. A estimativa de adolescentes obesos foi calculada em 7.187, com base nos alunos matriculados no ensino médio ${ }^{(14)}$ e com suporte em um estudo de base populacional de prevalência de obesidade em adolescentes brasileiros ${ }^{(17)}$.

Com esses valores, o tamanho da amostra ficou determinado em 232, no qual se acrescentou, aproximadamente, $17 \%$ para eventuais perdas de informações. Assim, foram avaliados 272 adolescentes, dos quais $73,5 \%$ eram das escolas públicas e $26,5 \%$, das particulares, mantendo proporção semelhante à distribuição de estudantes de Fortaleza, tendo sido pesquisadas todas as seis unidades regionais administrativas que compõem o Município, ressaltando a seleção de 47 escolas públicas e 17 escolas particulares.

As escolas, sorteadas de forma aleatória, receberam as orientações necessárias, sendo realizada a avaliação antropométrica dos alunos por membros habilitados da equipe de pesquisa. Em caso de não aceitação dos alunos ou da presença de um número reduzido de adolescentes obesos, novas escolas eram sorteadas naquela SER, a fim de completar o número de estudantes proposto para aquela região. Caso contrário, se o quantitativo de obesos excedesse o total determinado para a SER avaliada, era feito um sorteio simples para apontar os participantes da pesquisa.

Utilizaram-se como critérios de exclusão dos alunos: adolescentes pré-púberes e púberes, usuários de medicação que pudesse causar obesidade, deficientes físicos com limitação para ficar na posição ereta, grávidas, portadores de doenças genéticas e crônicas, com alteração no crescimento ponderal ou com limitação na postura, bem como os desnutridos ou eutróficos.

Aplicou-se um questionário semi-estruturado com os adolescentes obesos de escolas públicas e particulares, contendo dados de identificação, dados antropométricos (peso e altura), situação escolar, prática alimentar e nível de atividade física. As variáveis de exposição estudadas dividiram-se em sociodemográficas (sexo, idade e tipo de escola), de perfil alimentar (período da amamentação, horários regulares de refeições, realização do café da manhã, frequência semanal do consumo de alimentos fritos, fast food, carne gorda, doces, refrigerantes, frutas e verduras).

Coletaram-se dados antropométricos, peso e altura, obtidos em única tomada, com uso de balança digital, do modelo Plena, com capacidade para $180 \mathrm{~kg}$ e precisão de 100 g. A verificação da medida seguiu procedimento na qual o avaliado fica na posição ortostática, sobe cuidadosamente na balança e posiciona-se no seu centro. $\mathrm{O}$ aluno foi pesado descalço e com uniforme, devendo-se frisar que o uniforme foi descontado de acordo com cada escola, pelo fato de inexistir padronização. A estatura também foi avaliada por meio de tomada única, com antropômetro portátil, com precisão de $1 \mathrm{~cm}$, do tipo person-check da marca KaWe, fixado na parede com ponto zero no nível do solo. O avaliado permaneceu na posição ortostática, pés descalços e unidos, mantendo contato a fita com os calcanhares e com a região occipital ${ }^{(18)}$.

Utilizaram-se as medidas de peso e estatura para o cálculo do Índice de Massa Corporal (IMC), em que se dividiu o peso, em quilogramas, pelo quadrado da estatura, em metros $\left(\mathrm{IMC}=\right.$ peso/estatura $\left.{ }^{2}\right)$. Avaliou-se o IMC, considerando a idade do adolescente, tendo como parâmetro o adotado pela $\mathrm{OMS}^{(19)}$, o qual considera obesa a pessoa que tenha IMC $\geq$ percentil 97 . Em relação ao grau de obesidade, considerou-se como obesidade grave o escore $\mathrm{z}$ do $\mathrm{IMC}>3$, e moderada entre $\geq 2 \mathrm{e} \leq 3$, de acordo com a $\mathrm{WHO}^{(20)}$.

A avaliação da prática alimentar foi realizada por meio de um inquérito simplificado com o objetivo de caracterizar o hábito alimentar dos adolescentes obesos deste estudo. Vale salientar a utilização do inquérito simplificado, por ser uma investigação extensa, pesquisando vários fatores de risco para síndrome metabólica, não podendo ser feita, assim, uma avaliação mais específica. Esse tipo de abordagem, contudo, é permitida em estudos epidemiológicos. 
Para análise da prática alimentar que envolvia os alimentos e grupos de alimentos utilizou-se como parâmetro as diretrizes preconizadas pelo Guia Alimentar da População Brasileira $^{(21)}$, além de se basear em dois estudos ${ }^{(22,23)}$.

A fim de estimar o nível de atividade física, utilizouse um questionário internacional - International Physical Activity Questionnare - IPAQ, proposto pela Organização Mundial de Saúde, validado para uso em adolescentes na faixa etária deste ensaio ${ }^{(24)}$, consistindo em um questionário de avaliação de atividade física utilizado em muitos países e validado para emprego em adolescentes. Para análise mais simplificada, transformou-se o nível de atividade física em variável dicotômica, na qual se considerou os sedentários e os insuficientemente ativos como "pouca atividade" e os ativos e os muito ativos como "muita atividade".

Realizou-se a análise descritiva para caracterizar a distribuição da ocorrência dos eventos estudados, utilizandose o teste do qui-quadrado para avaliar as diferenças observadas nas prevalências entre os vários grupos, utilizando o programa Epi Info versão 6.04. Um valor de $p \leq 0,05$ foi considerado estatisticamente significante.

O Comitê de Ética do Complexo Hospitalar da Universidade Federal do Ceará (Protocolo COMEPE N ${ }^{\circ}$ 259/06. Of. $N^{\circ} 96 / 07$ ) aprovou o trabalho e os diretores das escolas autorizaram a pesquisa. Registrou-se o consentimento prévio, por escrito, dos adolescentes maiores de 18 anos e dos pais ou responsáveis dos menores de idade.

\section{RESULTADOS}

Observou-se que, dos 272 alunos obesos que compuseram o estudo, $112(41,2 \%)$ eram do sexo masculino e $160(58,8 \%)$ do feminino, com idade média de 16,5 anos $( \pm 1,31)$. Em relação à situação escolar, $73,5 \%(n=200)$ eram das escolas públicas e $26,5 \%(\mathrm{n}=72)$ de escolas privadas.

Não se constatou diferença significativa entre o grau de obesidade dos adolescentes de escolas públicas e privadas $(p>0,05)$. Observou-se maior prevalência da obesidade moderada nas escolas públicas $(85,5 \%, \mathrm{n}=171) \mathrm{e}$ particulares $(76,4 \%, n=55)$ (Figura 1).

Não houve diferença significativa entre ser amamentado exclusivamente até os seis meses de idade e o grau de obesidade em toda a população avaliada. Também não se observou essa associação, quando se avalia os alunos de colégios públicos $(\mathrm{p}=0,71)$ e os de particulares $(\mathrm{p}=0,96)$ (Figura 2).

Em relação às práticas alimentares, constatou-se 70,2\% $(n=192)$ dos adolescentes realizavam menos de cinco refeições ao dia. Não existindo diferença entre os alunos da $\mathrm{EPu}(71 \%, \mathrm{n}=142)$ e da $\operatorname{EPr}(68,1 \%, \mathrm{n}=49)(\mathrm{p}=0,63)$. O café da manhã não é uma prática comum entre os adolescentes

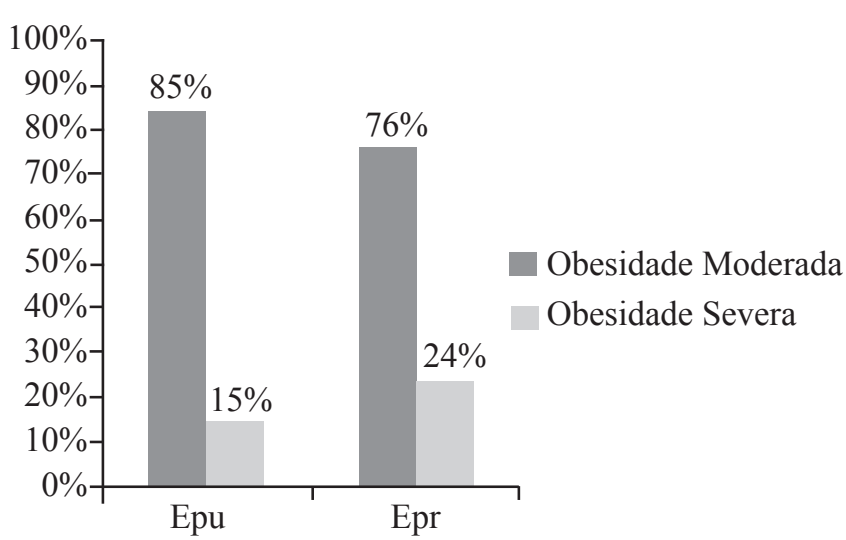

Figura 1 - Avaliação do grau de obesidade dos adolescentes de escolas públicas (EPu) e privadas (EPr). Fortaleza-CE, 2007-2009.

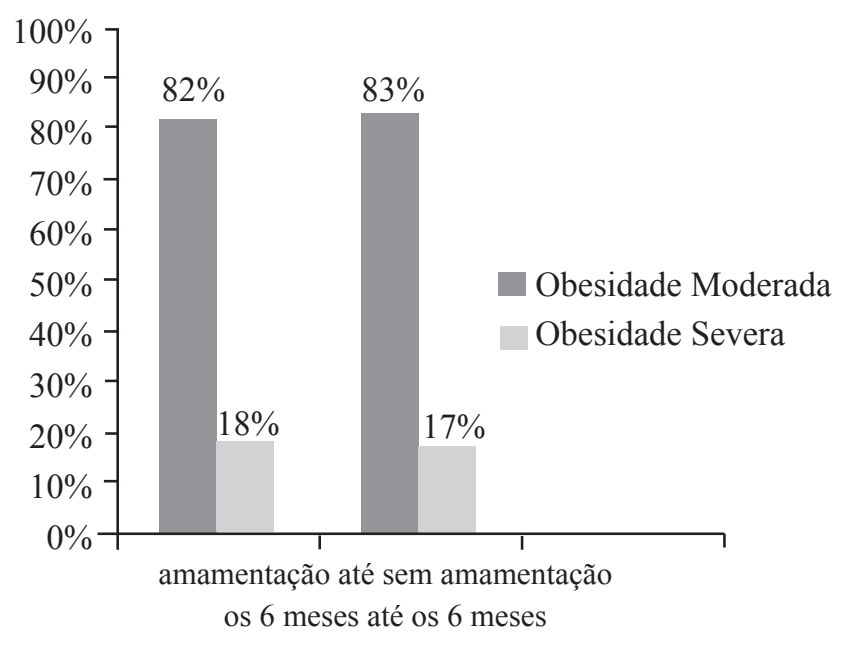

Figura 2 - Relação entre amamentação exclusiva (AME) até os seis meses de idade e o grau de obesidade nos adolescentes obesos. Fortaleza-CE, 2007-2009.

estudados, notadamente se considerarmos que 56,3\% $(\mathrm{n}=153)$ não realizavam essa refeição diariamente. Devese ressaltar que não se evidenciou diferença na realização dessa refeição entre os adolescentes de $\mathrm{EPu}(56 \%, \mathrm{n}=112) \mathrm{e}$ de $\operatorname{EPr}(56,9 \%, \mathrm{n}=41 ; \mathrm{p}=0,88)$.

Dos adolescentes estudados, $70,0 \%(n=190)$ não faziam as refeições nos mesmos horários que os familiares. Não foi apontada diferença significativa $(\mathrm{p}=0,61)$ em relação a essa atitude, entre os jovens avaliados da $\mathrm{EPu}(69 \%, \mathrm{n}=138) \mathrm{e}$ da $\operatorname{EPr}(72,2 \%, \mathrm{n}=52)$.

Observou-se que $30 \% \quad(\mathrm{n}=82)$ dos adolescentes ingeriam as refeições diárias na mesa com a família. Houve diferença significativa nos grupos avaliados $(\mathrm{p}=0,02)$, pois se constatou que os estudantes dos colégios privados 
$(47,2 \%, n=34)$ realizavam as refeições mais com a família do que os dos públicos $(32 \%, n=64)$. Não se observou associação entre o hábito de não fazer as refeições com a família e o grau de obesidade $(\mathrm{p}=0,52)$.

Verificou-se que os 56,9\% (n=41) alunos da $\operatorname{EPr}$ consumiam mais refrigerantes ( $\geq 3$ dias da semana) quando comparados aos $41,5 \%(n=83)$ da $\mathrm{EPu}(\mathrm{p}=0,03)$, porém sem associação entre consumo de refrigerantes e grau da obesidade $(\mathrm{p}=0,19)$.

Somente $10 \% \quad(n=27)$ dos adolescentes avaliados comiam frutas diariamente. Não se encontrou diferença entre os da $\mathrm{EPu}(9,5 \%, \mathrm{n}=19)$ e da $\operatorname{EPr}(11,3 \%, \mathrm{n}=8$; $\mathrm{p}=0,67$ ).

Constatou-se $14,1 \% \quad(n=38)$ consumiam vegetais diariamente, não observando diferença significativa entre os alunos dos colégios públicos $(15,1 \%, \mathrm{n}=30)$ e dos particulares $(11,4 \%, n=8 ; \mathrm{p}=0,45)$.

No que diz respeito ao consumo de comidas fritas na semana, houve diferença entre aqueles que apresentavam baixo consumo deste alimento. Nesse grupo, havia 12,5\% $(n=25)$ dos alunos das escolas públicas e 4,2\% (n=3) das particulares, com diferença significativa $(p=0,04)$. Destaque-se a falta de associação entre baixo consumo de comidas fritas pelos alunos da EPr e da EPu com o grau de obesidade $(\mathrm{p}=0,45)$.

$\mathrm{O}$ alto consumo de comidas fritas (acima de 5 dias na semana) foi detectado em 23,5\% ( $=47)$ dos alunos da EPu e em 20,8\% (n=15) da EPr, não tendo sido encontrada relação significativa entre este consumo e o grau de obesidade $(\mathrm{p}=0,64)$.

Dos adolescentes estudados, $47 \%(\mathrm{n}=94)$ dos alunos da EPu e 32,4\% (n=23) da EPr não consumiam fast-food, mostrando diferença significativa $(\mathrm{p}=0,02)$. Vale considerar que, dentre os alunos que não comiam fast-food, não foi apontada diferença significante entre os das escolas públicas e particulares com o grau de obesidade $(\mathrm{p}=0,64)$.

Quanto ao consumo elevado (acima de 5 dias na semana) comprovou-se que 7\% $(n=14)$ dos alunos da $\mathrm{EPu}$ e 7\% $(n=5)$ da EPr tinham essa prática inadequada, sem diferença estatisticamente significante $(\mathrm{p}=0,98)$.

Salienta-se não haver diferença significativa na frequência do consumo semanal de doces $(p=0,43)$, chocolates $(p=0,95)$ e carnes gordas $(p=0,68)$ entre os alunos de escolas públicas e particulares. Além disso, não se observou consumo exagerado desses alimentos na maioria dos adolescentes (figura 3).

Com relação à atividade física, verificou-se que $71 \%$ $(\mathrm{n}=193)$ praticavam muita atividade física (ativos e muito ativos) e $29 \%(n=79)$ realizavam pouca atividade física (sedentários e insuficientemente ativos), de acordo com o IPAQ. A tabela I exibe a associação da prática de atividade física entre os estudantes das redes de ensino. Identificou-se o fato de que os adolescentes obesos das escolas públicas são mais ativos do que os das particulares $(p=0,0002)$. Deve-se ressaltar que não se apontou diferença significante entre o nível de atividade física e o grau de obesidade $(\mathrm{p}=0,88)$.

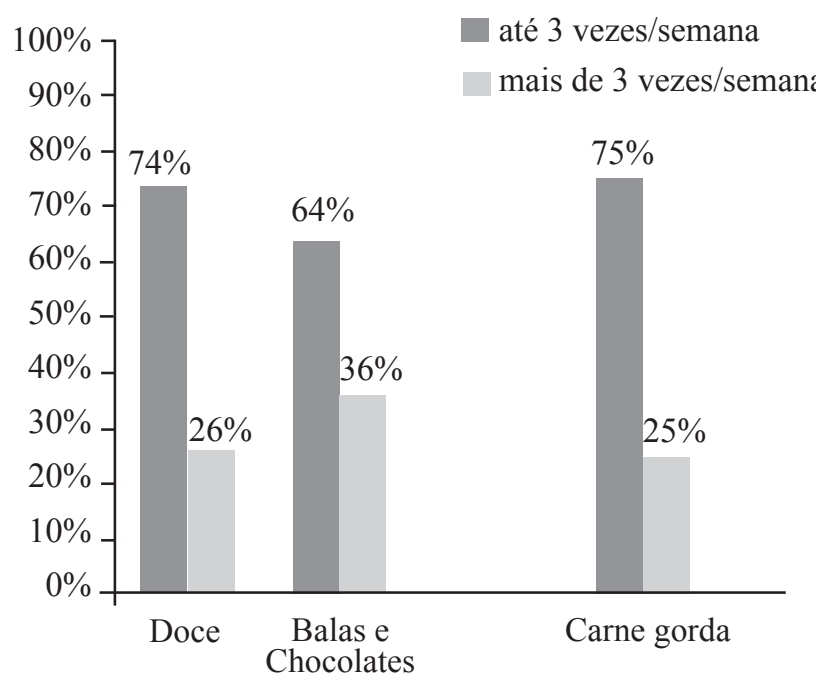

Figura 3 - Frequência do consumo semanal de doces, balas e chocolates, e carnes gordas da população estudada. Fortaleza-CE, 2007-2009.

Tabela I - Comparação dos adolescentes obesos de escolas públicas e privadas quanto ao nível de atividade física (AF) praticada. Fortaleza-CE, 2007-2009.

\begin{tabular}{lccc}
\hline \multirow{2}{*}{ Variável } & \multicolumn{2}{c}{ Nível de atividade física } & \\
\cline { 2 - 3 } & $\begin{array}{c}\text { Adolescente pouco ativo } \\
(\mathbf{n} ; \%)\end{array}$ & $\begin{array}{c}\text { Adolescente muito ativo } \\
\mathbf{( n ;} \boldsymbol{\%})\end{array}$ \\
\hline Escola & $55(77,0 \%)$ & $17(23,0 \%)$ & 0,0002 \\
Privada & $108(54,2 \%)$ & $92(45,8 \%)$ & \\
Pública & & & \\
\hline
\end{tabular}




\section{DISCUSSÃO}

No Brasil, existem poucos estudos de base populacional sobre hábitos alimentares e atividade física, particularmente em adolescentes ${ }^{(25)}$. Este estudo é um dos poucos relacionados à obesidade nessa faixa etária. Além disso, representa amostra probabilística, pois a população do estudo foi tomada aleatoriamente, representando as diversas classes sociais e todas as regiões da cidade de Fortaleza.

Observou-se o fato de não existir diferença entre os adolescentes obesos de escolas públicas e particulares no que se refere ao grau de obesidade (de moderado e severo), havendo, assim, maior predomínio de obesos moderados, tanto nas escolas públicas quanto nas particulares. Essa constatação demonstra que as diferenças sociais não influenciam na intensidade da obesidade.

Estudo realizado em Recife, comparando a prevalência de obesidade em adolescentes de variadas condições socioeconômicas, observou, em relação à obesidade, uma maior prevalência nos adolescentes de alta renda ${ }^{(26)}$. É importante ressaltar que a maioria das pesquisas avalia a prevalência da obesidade em adolescentes entre as diversas classes sociais, não sendo investigado nesses grupos o grau de obesidade, como foi realizado neste estudo.

O estímulo ao aleitamento materno é um dos fatores de proteção e prevenção contra a obesidade, pois pesquisas mostram as crianças não amamentadas ou com menor tempo de amamentação com maior risco de ficarem obesas durante a vida(27). Esta pesquisa fez a comparação entre os adolescentes obesos, não podendo ser cotejado com a maioria dos estudos da literatura, que comparam os adolescentes eutróficos com os obesos, no que concerne à amamentação ${ }^{(27)}$. Observou-se nesta pesquisa que a amamentação exclusiva até seis meses não tinha associação com o grau de obesidade.

Vale salientar que os adolescentes são muitas vezes considerados um grupo exposto ao risco nutricional, em razão de hábitos alimentares inadequados. Neste estudo, pôde-se observar que os adolescentes obesos possuem o costume de omitir o café da manhã, sendo esse mesmo resultado observado em outras análises ${ }^{(28)}$. Consoante com este achado, crianças sem o hábito de tomar café da manhã estão mais susceptíveis à obesidade e este fato pode ser associado a várias causas ${ }^{(29)}$.

O consumo do café da manhã exerce controle sobre a fome, o apetite e alguns hormônios, propiciando adequado controle glicêmico e lipídico. Os alimentos normalmente consumidos nessa refeição (frutas, laticínios e cereais integrais) são fontes de fibras alimentares que controlam a absorção de outros alimentos e, dessa maneira, os mecanismos de fome e saciedade. Outro fator importante a ser destacado é que a ausência dessa refeição pode dificultar a elevação da glicemia adequada às atividades matinais e acarretar uma deficiência de cálcio ${ }^{(29)}$.

A maioria dos jovens analisados nesta pesquisa não tinha regularidade nos horários das refeições. Não se evidenciou diferença entre os alunos da rede pública e do sistema particular. A ausência de regularidade no horário das refeições é um fator que contribui para o desenvolvimento da obesidade ${ }^{(30)}$.

Foi observado que a maioria dos jovens analisados não fazia as refeições com os familiares. Estudos demonstram associação positiva entre realizar refeições com a família e a ingestão de alimentos saudáveis, sendo esse comportamento um fator protetor para o excesso de peso $^{(31)}$. Na população avaliada, evidenciou-se o hábito de não realizar refeições com a família era mais frequente nos alunos das escolas públicas, contudo, essa diferença não repercutiu no grau de obesidade entre os grupos estudados.

No que diz respeito ao consumo de refrigerantes, os obesos da rede particular apresentaram maior consumo quando comparados com os da rede pública, dado esse também encontrado em outros estudos ${ }^{(32,33)}$. É importante relatar a elevada densidade energética dos refrigerantes, resultante do alto conteúdo de açúcar, fornecendo, dessa maneira, calorias vazias. Além disso, por serem produtos sob forma líquida, não ativam os centros de saciedade, levando o indivíduo a ter maior ingestão energética. Portanto, os refrigerantes podem ser considerados corresponsáveis pelo ganho de peso $^{(34)}$.

Corroborando com o presente estudo, outros trabalhos mostraram a reduzida ingestão de frutas e verduras é frequente entre adolescentes ${ }^{(4,32,33)}$. Em investigação anteriormente realizada, mostrou associação entre o consumo reduzido de legumes e verduras com a presença de sobrepeso e obesidade nos jovens da classe econômica $\mathrm{B}^{(33)}$. Esses resultados, no entanto, estão diferentes dos encontrados nesta pesquisa, uma vez que não foi evidenciada diferença nesse hábito alimentar, entre os adolescentes obesos de escolas públicas e privadas.

O consumo insuficiente de frutas, verduras e legumes é um fator de risco relacionado à causa de doenças crônicas não transmissíveis para a população. Esses alimentos são importantes para uma dieta saudável, pois são fontes de micronutrientes, fibras e outros componentes com propriedades funcionais. As frutas e hortaliças têm baixa densidade energética, o que favorece a manutenção saudável do peso corporal. Assim, é muito importante que o adolescente tenha um consumo adequado desses alimentos ${ }^{(35)}$. Estudo realizado com adolescentes na cidade de São Paulo(4) corrobora com os resultados encontrados nesta pesquisa, quando acentuou o padrão alimentar dos adolescentes participantes caracterizado por baixa ingestão 
de frutas e vegetais. Portanto, a tendência atual apresentada pelos adolescentes no consumo alimentar constitui motivo de preocupação.

De acordo com as pesquisas, o consumo de fast foods aumenta o desenvolvimento da obesidade e das comorbidades associadas, sendo essa refeição rica em calorias, gorduras saturadas e sal; bem como deficiente de vitaminas, minerais e fibras. Quando o fast food faz parte da rotina, acarretará o aumento de peso e nutrição inadequada ${ }^{(36)}$. Neste artigo, pode-se observar a prevalência de adolescentes obesos de escolas públicas que nenhuma vez consomem fast food na semana é maior do que a das escolas privadas.

A maioria dos estudos ${ }^{(1,37)}$ aponta que os adolescentes, tanto obesos quanto eutróficos, consomem em excesso alimentos de baixo valor nutritivo e alta densidade calórica, tais como, doces, balas e chocolates, ao passo que, na presente pesquisa, constatou-se os adolescentes obesos de escolas públicas e privadas consomem uma quantidade moderada desses alimentos.

Acredita-se que independente da classe socioeconômica, a prática esportiva em horários de lazer não mostra associação com a presença do sobrepeso e obesidade ${ }^{(33)}$. Esse fato foi encontrado neste estudo e em outros relatos da literatura ${ }^{(4,38)}$.

Estudo com 1.229 adolescentes de escolas públicas e privadas, sendo esses classificados em insuficientemente ativos $(22,3 \%)$ e muito ativos $(77,7 \%)$, observou que os alunos da rede pública são mais ativos se comparados com os da rede particular. Vislumbrou-se ausência de diferença significativa entre o nível de atividade física e índice de massa corporal ${ }^{(39)}$. Tais conclusões são similares a este estudo, embora a amostra de jovens avaliados tenha sido somente de obesos.

O hábito de realizar atividade física na adolescência traz vários benefícios para a saúde, como controle e manutenção do peso corporal e redução de riscos cardiovasculares. Os dados desta pesquisa indicaram que a maioria dos adolescentes obesos eram ativos, não estando em consonância com estudos da literatura ${ }^{(40)}$. Isso pode ser justificado pela característica do delineamento do estudo, que, sendo transversal, possibilita a ocorrência de haver a causalidade reversa, na qual os adolescentes obesos poderiam ter adquirido o hábito de atividade física para redução do peso.

Portanto, devem-se realizar medidas de caráter educativo e informativo sobre o incentivo à aquisição de hábitos alimentares saudáveis, como a inclusão de alimentos in natura, principalmente frutas e verduras, e redução de açúcares simples, ações que devem ser praticadas. Como foram encontradas poucas diferenças entre os hábitos alimentares dos alunos de escolas públicas e particulares, faz- se necessária uma intervenção imediata, com uma reeducação alimentar nos adolescentes, independentemente da classe socioeconômica a que esses jovens pertençam, porém considerando a individualidade e realidade de cada grupo.

Deve-se ressaltar o fato de que a principal limitação do estudo foi o pequeno número de trabalhos científicos com avaliações similares, dificultando as comparações. Apresenta, contudo, dados pouco estudados e que são importantes na avaliação dos jovens obesos. Observouse também a utilização de um inquérito alimentar simplificado com baixa precisão na análise, que, apesar de pouco adequado, era o mais apropriado para este tipo de avaliação. Também pode ter ocorrido o viés recordatório, principalmente dos dados do início da vida, como amamentação.

\section{CONCLUSÃO}

Os adolescentes obesos apresentam prática alimentar inadequada e há poucas diferenças desses hábitos entre os alunos das escolas públicas e particulares. Em relação à atividade física, os jovens avaliados das escolas públicas são mais ativos do que os do sistema privado, mas essa diferença não influenciou no grau de obesidade entre os grupos estudados.

\section{AGRADECIMENTOS}

À direção das Instituições, que permitiram a realização da pesquisa em suas dependências, e aos alunos que compreenderam os objetivos da pesquisa e se dispuseram a contribuir com as suas participações.

\section{REFERÊNCIAS}

1. Marchi-Alves LM, Yagui CM, Rodrigues CS, Mazzo A, Rangel EML, Girão FB. Obesidade infantil ontem e hoje: importância da avaliação antropométrica pelo enfermeiro. Esc Anna Nery Rev Enferm. 2011;15(2):238-44.

2. Azevedo FR, Brito BC. Influência das variáveis nutricionais e da obesidade sobre a saúde e o metabolismo. Rev Assoc Med Bras. 2012;58(6):71423.

3. Cavalcanti CBS, Carvalho SBCE, Barros MVG. Indicadores antropométricos de obesidade abdominal: revisão dos artigos indexados na biblioteca SciELO. Rev Bras Cineantropom Desempenho Hum. 2009;11(2):217-25. 
4. Murillo OO, Rey MCPA. Panorama de prácticas de alimentación de adolescentes escolarizados. Av Enferm. 2009;27(2):43-56.

5. Wanderley EM, Ferreira VA. Obesidade: uma perspectiva plural. Ciênc Saúde Coletiva. 2010;15(1):185-94.

6. Cardoso CBMA, D'abreu HCC, Ribeiro MG, Bouzas RI. Obesidade na adolescência: reflexões e abordagem. Adolesc Saude. 2010;7(1):12-8.

7. Lamounier JA, Welford VRS, Parizzi MR, Lamounier FB. Obesidade na Adolescência. In: Priore SE, Oliveira RMS, Faria ER, Franceschini SCC, Pereira PF. Nutrição e Saúde na Adolescência. Rio de Janeiro: Editora Rubio; 2010. p. 75-92.

8. Suplicy HL. Obesidade: tratamento dietético. In: Associação Brasileira para o Estudo da Obesidade e Síndrome Metabólica. Diretrizes brasileiras de obesidade 2009/2010 / ABESO. $3^{a}$ ed. São Paulo: AC Farmacêutica; 2009. p. 33-41.

9. Instituto Brasileiro de Geografia e Estatística IBGE. Pesquisa de Orçamento Familiar. Análise de Disponibilidade Domiciliar de Alimentos e do Estado Nutricional no Brasil: 2008-2009. Rio de Janeiro: IBGE; 2010. p. 1-7.

10. Souza EB. Transição nutricional no Brasil: análise dos principais fatores. Cad Unifoa [periódico na internet]. 2010 [acesso em 2012 Set 8];5(13):49-53. Disponível em: http://web.unifoa.edu.br/cadernos/edicao/13/49.pdf.

11. Mathus-Vliegen L, Toouli J, Fried M, Khan AG, Garisch J, Hunt R, et al. Obesity. World Gastroenterology Organization Global Guidelines [periódico na internet]. 2011 [acesso em 2012 Set 8]:1-17. Disponível em: http://www.worldgastroenterology.org/assets/export/ userfiles/Obesity-Master\%20Document $\% 20$ for $\% 20$ Website.pdf.

12. Casazza K, Dulin-Keita A, Gower BA, Fernandez JR. Differential influence of diet and physical activity on components of metabolic syndrome in a multiethnic sample of children. J Am Diet Assoc. 2009;109(2):23644.

13. Campos LA. Prevalência e Fatores Associados à Síndrome Metabólica em Adolescentes Escolares Obesos do Município de Fortaleza [tese]. Fortaleza: Universidade Federal do Ceará Faculdade de Medicina; 2011.

14. Governo do Estado do Ceará (BR), Secretaria de Educação. Estatísticas da Educação Básica nos
Municípios do Ceará [acesso em 2010 Jan 20]. Disponível em: http://download.seduc.ce.gov.br/ indicadores/fortaleza.pdf.

15. Siervogel RM, Demerath EW, Schubert C, Remsberg KE, Chumlea WC, Sun S, et al. Puberty and body composition. Horm Res. 2003;60(Suppl 1):36-45.

16. Duncan GE, Li SM, Zhou XH. Prevalence and trends of a metabolic syndrome phenotype among U.S. Adolescents, 1999-2000. Diabetes Care. 2004;27(10):2438-43.

17. Terres NG, Pinheiro RT, Horta BL, Pinheiro KAT, Horta LL. Prevalence and factors associated to overweight and obesity in adolescents. Rev Saúde Pública. 2006;40(4):627-33.

18. Waitzberg DL, Ferrini MT. Exame Físico e Antropometria. In: Waitzberg DL. Nutrição oral, enteral e parenteral na prática clínica. $3^{\mathrm{a}}$ ed. São Paulo: Atheneu; 2000. p. 255-78.

19. World Health Organization. Obesity: preventing and managing the global epidemic. Geneva: World Health Organization; 2000.

20. Onis M, Onyango AW, Borghi E, Siyam A, Nishida C, Siekmann J. Development of a WHO growth reference for school-aged children and adolescents. Bull World Health Organ. 2007;85(9):660-7.

21. Ministério da Saúde (BR), Secretaria de Atenção à Saúde, Coordenação Geral da Política de Alimentação e Nutrição. Guia Alimentar para população brasileira: promovendo a alimentação saudável. Brasília: Ministério da Saúde; 2008.

22. Silveira D, Taddei JA, Escrivão MA, Oliveira FL, Ancona-Lopez F. Risk factors for overweight among Brazilian adolescents of low-income families: a casecontrol study. Public Health Nutr. 2006;9(4):421- 8.

23. Neutzling MB, Taddei JA, Gigante DP. Risk factors of obesity among Brazilian adolescents: a case-control study. Public Health Nutr. 2003;6(8):743- 49.

24. Hagströmer M, Bergman P, De Bourdeaudhuij I, Ortega FB, Ruiz JR, Manios Y, et al. Concurrent validity of a modified version of the International Physical Activity Questionnaire (IPAQ-A) in European adolescents: The HELENA Study. Int J Obes. 2008;32(Suppl 5):S42-8.

25. Neutzling MB, Araújo CLP, Vieira MFA, Hallal PC, Menezes AMB. Freqüência de consumo de dietas ricas em gordura e pobres em fibra entre adolescentes. Rev Saúde Pública. 2007;41(3):336-42. 
26. Silva GAP, Balaban G, Motta MEFA. Prevalência de sobrepeso e obesidade em crianças e adolescentes de diferentes condições socioeconômicas. Rev Bras Saúde Matern Infant. 2005;5(1):53-9.

27. Siqueira RS, Monteiro CA. Amamentação na infância e obesidade na idade escolar em famílias de alto nível socioeconômico. Rev Saúde Pública. 2007;41(1):5-12.

28. Binn Zaal AA, Musaiger AO, D'Souza R. Dietary habits associated with obesity among adolescents in Dubai, United Arab Emirates. Nutr Hosp. 2009;24(4):437-44.

29. Höfelmann DA, Momm N. Breakfast: omission and associated factors in schoolchildren from Itajaí, Santa Catarina state, Brazil. Nutrire Rev Soc Bras Aliment Nutr. 2014;39(1):40-55.

30. Ministério da Saúde (BR), Secretaria de Atenção à Saúde, Departamento de Atenção Básica. Obesidade. Brasília: Ministério da Saúde; 2006. (Cadernos de Atenção Básica, n. 12).

31. Cardoso LO, Engstrom EM, Leite IC, Castro IRR. Fatores socioeconômicos, demográficos, ambientais e comportamentais associados ao excesso de peso em adolescentes: uma revisão sistemática da literatura. Rev Bras Epidemiol. 2009;12(3):378-403.

32. Ceschini FL, Andrade DR, Oliveira LC, Araújo Júnior JF, Matsudo VKR. Prevalence of physical inactivity and associated factors among high school students from state's public schools. J Pediatr. 2009;85(4):301-6

33. Lippo BRS, Silva IM, Aca CRP, Lira PIC, Silva GAP, Motta MEFA. Determinants of physical inactivity among urban adolescents. J Pediatr. 2010;86(6):520-4.

34. Bessa $\mathrm{M}$, Valente $\mathrm{H}$, Cordeiro T, Padrão $\mathrm{P}$, Moreira $\mathrm{A}$, Lopes $\mathrm{C}$, et al. Ingestão de alimentos fluidos e risco de excesso de peso em crianças. Acta Med Port. 2008;21(2):161-70.
35. Mendes KL, Catão LP. Avaliação do consumo de frutas, legumes e verduras por adolescentes de Formiga - MG e sua relação com fatores socioeconômicos. Alim Nutr. 2010;21(2):291-6.

36. Jamie S. Nutrição na Adolescência. In: Mahan LK, Escott-Stump S. Krause, alimentos, nutrição e dietoterapia. $12^{\mathrm{a}}$ ed. Rio de Janeiro: Elsevier; 2010. p. 255-56.

37. Leal GVS, Philippi ST, Matsudo SMM, Toassa EC. Consumo alimentar e padrão de refeições de adolescentes, São Paulo, Brasil. Rev Bras Epidemiol. 2010;13(3):457-67.

38. Reichert FF, Baptista Menezes AM, Wells JCK, Carvalho Dumith S, Hallal PC. Physical activity as a predictor of adolescent body fatness: a systematic review. Sports Med. 2009;39(4):279-94.

39. Silva RCD. Obesidade, perfil lipídico e sua relação com o nível de atividade física de adolescentes escolares [dissertação]. Brasília: Universidade de Brasília; 2007.

40. Enes CC, Slater B. Obesidade na adolescência e seus principais fatores determinantes. Rev Bras Epidemiol. 2010;13(1):163-71.

\section{Endereço para correspondência:}

Lívia Freitas Campos

Avenida Antônio Justa, 3300/402

Bairro: Meireles

CEP: 60165-090 - Fortaleza - CE - Brasil

E-mail: liviafreitas_@hotmail.com 